\title{
On certain regularity properties of Haar-null sets
}

\author{
by \\ Pandelis Dodos (Athens)
}

\begin{abstract}
Let $X$ be an abelian Polish group. For every analytic Haar-null set $A \subseteq X$ let $T(A)$ be the set of test measures of $A$. We show that $T(A)$ is always dense and coanalytic in $P(X)$. We prove that if $A$ is compact then $T(A)$ is $G_{\delta}$ dense, while if $A$ is non-meager then $T(A)$ is meager. We also strengthen a result of Solecki and we show that for every analytic Haar-null set $A$, there exists a Borel Haar-null set $B \supseteq A$ such that $T(A) \backslash T(B)$ is meager. Finally, under Martin's Axiom and the negation of Continuum Hypothesis, some results concerning co-analytic sets are derived.
\end{abstract}

1. Introduction and auxiliary lemmas. A universally measurable subset $A$ of an abelian Polish group $X$ is called Haar-null if there exists a probability Borel measure $\mu$ on $X$, called a test measure of $A$, such that $\mu(x+A)=0$ for every $x \in X$. This definition is due to J. P. R. Christensen [3] and extends the usual notion of a Haar-measure zero set. The complement of a Haar-null set is called prevalent. The class of Haar-null sets has also been considered by Hunt, Sauer and Yorke in [5]. They used the term shy instead of Haar-null.

If $X$ is locally compact, then a universally measurable set $A$ is Haar-null if and only if there exists a Borel Haar-null set $B \supseteq A$. In the non-locally compact case the situation is different. As R. Dougherty observed in [4], answering a problem of Mycielski (see [9]), assuming Continuum Hypothesis (or just Martin's Axiom) there exists a universally measurable set $A$ which is not contained in any Borel Haar-null set. However S. Solecki (see [11], [12]) proved that if $A$ is an analytic Haar-null set, then this can be done.

In this paper we are concerned with the properties of the set of test measures $T(A)$ of an analytic Haar-null set $A$. We show that this set is always dense and co-analytic in $P(X)$ (by $P(X)$ we denote the space of all probability Borel measures on $X$ endowed with the weak topology). If $A$ is not meager and Haar-null, then we prove that $T(A)$ is meager in $P(X)$. On the other hand we show that compact sets are tested by co-meager many measures.

2000 Mathematics Subject Classification: 28C10, 28A05, 43A05.

Research supported by a grant of EPEAEK program "PYTHAGORAS". 
We also strengthen the above-mentioned result of Solecki. We prove that for every analytic Haar-null set $A$, there exists a Borel Haar-null set $B \supseteq A$ such that $T(A) \backslash T(B)$ is meager in $P(X)$. That is, almost every test measure for $A$ is a test measure for $B$. Actually, we prove a general theorem which permits us to derive, besides the just mentioned result, an approximation type result for analytic sets. The proof of this theorem is combinatorial in nature.

Under stronger set-theoretic assumptions, in particular under Martin's Axiom and the negation of Continuum Hypothesis, we show that $\boldsymbol{\Pi}_{2}^{1}$ sets still behave well at least with respect to translations over co-meager sets. We also derive a reduction type result for analytic and co-analytic sets. Again, under Martin's Axiom and the negation of Continuum Hypothesis, we show that for every analytic or co-analytic set $A \subseteq X$ there exists a Borel set $B \subseteq A$ such that $T(B) \backslash T(A)$ is meager in $P(X)$. Finally, an application of these ideas is given in the last section, in the theory of essentially smooth Lipschitz functions.

Notations. In what follows, for any Polish space $X$, by $P(X)$ we denote (as we have already mentioned) the space of all Borel probability measures on $X$. The set $P(X)$ equipped with the weak topology becomes a Polish space (see for instance [7, p. 112]). If $d$ is a compatible complete metric for $X$, then a compatible complete metric for $P(X)$ is the so-called Lévy metric $\varrho$, defined by

$$
\varrho(\mu, \nu)=\inf \left\{\delta \geq 0: \mu(A) \leq \nu\left(A_{\delta}\right)+\delta \text { and } \nu(A) \leq \mu\left(A_{\delta}\right)+\delta\right\}
$$

where $A_{\delta}=\{x \in X: d(x, A) \leq \delta\}$. For every $\mu \in P(X)$ by supp $\mu$ we denote the support of $\mu$. All the other pieces of notation we use are standard (for more information we refer to [7]).

Some auxiliary lemmas. Let $X$ be an abelian Polish group. Given $\mu \in$ $P(X)$ and $x \in X$, we define the measure $\mu_{x} \in P(X)$ by $\mu_{x}(A)=\mu(x+A)$ for every Borel subset $A$ of $X$.

Lemma 1. (i) For every $\mu \in P(X)$, the function $x \mapsto \mu_{x}$ is continuous.

(ii) The function $(\mu, x) \mapsto \mu_{x}$ is (jointly) continuous.

Proof. Part (i) follows from Lebesgue's dominated convergence theorem, while (ii) follows from Theorem 9.14 in [7] and (i).

LemMA 2. Let $\varepsilon \geq 0$. Then:

(i) If $F \subseteq X$ is closed, then the set $\{(\mu, x) \in P(X) \times X: \mu(x+F) \geq \varepsilon\}$ is closed in $P(X) \times X$.

(ii) If $B \subseteq X$ is Borel and $\mu \in P(X)$, then the set $\{x \in X: \mu(x+B)>\varepsilon\}$ is Borel in $X$. 
Proof. (i) Recall that if $F \subseteq X$ is closed, then the function $\mu \mapsto \mu(F)$ is upper semicontinuous (see $[7$, p. 111]). As the composition of a continuous function with an upper semicontinuous real-valued function is upper semicontinuous, the result follows by Lemma 1(ii).

(ii) We recall that if $B \subseteq X$ is Borel, then the function $\mu \mapsto \mu(B)$ is Borel measurable (see [7, p. 112]). So the result follows by Lemma 1(i).

2. The set of test measures. In this section $X$ will be an uncountable abelian Polish group (locally or non-locally compact). For every universally measurable set $A \subseteq X$ we put

$$
T(A)=\{\mu \in P(X): \mu(x+A)=0 \text { for every } x \in X\} .
$$

That is, $T(A)$ is the set of all test measures of $A$. Clearly $A$ is Haar-null if and only if $T(A) \neq \emptyset$. The following simple fact will be useful in what follows. The proof is trivial.

LEMMA 3. Let $\left(A_{n}\right)_{n}$ be a sequence of universally measurable subsets of $X$. Then $T(A)=\bigcap_{n} T\left(A_{n}\right)$, where $A=\bigcup_{n} A_{n}$.

We have the following estimate for the complexity of the set of test measures of an analytic set.

Lemma 4. If $A \subseteq X$ is analytic, then $T(A)$ is a co-analytic subset of $P(X)$.

Proof. By Theorem 29.26 in [7], the set $\{(\mu, x) \in P(X) \times X: \mu(x+A)$ $=0\}$ is $\Pi_{1}^{1}$ and the lemma follows.

REMARK 1 . If $A$ is a co-analytic subset of $X$, then it is also easily verified that the set $T(A)$ is $\boldsymbol{\Pi}_{2}^{1}$.

In the following proposition we state some properties of the set of test measures.

Proposition 5. Let $A \subseteq X$ be a universally measurable Haar-null set. Then:

(i) $T(A)$ is always dense in $P(X)$.

(ii) If $A$ is analytic non-meager, then $T(A)$ is meager.

(iii) If $A$ is $\sigma$-compact, then $T(A)$ is co-meager.

Proof. (i) Fix a compatible complete metric $d$ for $X$. For $x \in X$ and $r \geq 0$, we put $B(x, r)=\{y \in X: d(x, y) \leq r\}$. Recall that if $D$ is a countable dense subset of $X$, then the set of all convex combinations of Dirac measures $\left(\delta_{x}\right)_{x \in D}$ is dense in $P(X)$ (see [7, p. 110]). So let $\nu=\sum_{i=1}^{l} a_{i} \delta_{x_{i}}$ and $r>0$, where $x_{i} \in D$ and $\sum_{i=1}^{l} a_{i}=1$, with $a_{i}>0$. We will find $\mu \in T(A)$ with $\varrho(\nu, \mu)<r$. As $r$ and $\nu$ were arbitrary, this will finish the proof. Let $r^{\prime}>0$ be small enough so that $r^{\prime}<r$ and $B\left(x_{i}, r^{\prime}\right) \cap B\left(x_{j}, r^{\prime}\right)=\emptyset$ 
for every $i, j \in\{1, \ldots, l\}$ with $i \neq j$. It is easy to verify that for every $i \in\{1, \ldots, l\}$ we may find a measure $\mu_{i} \in T(A)$ such that $x_{i} \in \operatorname{supp} \mu_{i}$ and supp $\mu_{i} \subset B\left(x_{i}, r^{\prime}\right)$. Put $\mu=\sum_{i=1}^{l} a_{i} \mu_{i}$. Then $\varrho(\nu, \mu)<r$ and clearly $\mu \in T(A)$.

(ii) Let $\left(x_{n}\right)_{n}$ be a countable dense subset of $X$ and put $B=\bigcup_{n}\left(x_{n}+A\right)$. Then $B$ is co-meager in $X$ and moreover $T(A)=T(B)$. Pick a dense $G_{\delta}$ subset $G$ of $B$. Note that $T(A) \subseteq P(X) \backslash\{\mu \in P(X): \mu(G)=1\}$. As $\{\mu \in P(X): \mu(G)=1\}$ is co-meager (see [7]), the result follows.

(iii) By Lemma 3, it is enough to show that if $K \subseteq X$ is compact, then $T(K)$ is co-meager. We already know from (i) that $T(K)$ is dense in $P(X)$. We will show that it is also $G_{\delta}$. Let

$$
A=\{\mu \in P(X): \exists x \in X \text { such that } \mu(x+K)>0\} .
$$

Also, for every $m \geq 1$, let

$$
A_{m}=\{\mu \in P(X): \exists x \in X \text { such that } \mu(x+K)>1 / m\} .
$$

Clearly $A=\bigcup_{m} A_{m}$. Then observe that $T(K)=P(X) \backslash A$. We will show that for every $m$ we have $\bar{A}_{m} \subseteq A$. This implies that $A=\bigcup_{m} \bar{A}_{m}$ and in particular that $T(K)$ is $G_{\delta}$.

Fix $m$ and let $\left(\mu_{n}\right)_{n} \subseteq A_{m}$ with $\mu_{n} \rightarrow \mu$ in $P(X)$. We will show that $\mu \in A$. As $\mu_{n} \in A_{m}$, for every $n$ there exists an $x_{n} \in X$ such that $\mu_{n}\left(x_{n}+K\right)$ $>1 / m$. Put $D=\left(x_{n}\right)_{n}$. We distinguish two cases.

First assume that $\bar{D}=D$. In this case $D$ is countable and closed. Put $F=\bigcup_{n}\left(x_{n}+K\right)$. Then, as $K$ is compact, $F$ is a closed subset of $X$. Also note that for every $n$ we have $\mu_{n}(F) \geq \mu_{n}\left(x_{n}+K\right)>1 / m$. As $\mu_{n} \rightarrow \mu$ and $F$ is closed, we get

$$
\mu(F)=\lim \sup \mu_{n}(F) \geq 1 / m>0 .
$$

From the fact that $F$ is the countable union of the sets $x_{n}+K$ we conclude that there exists an $n$ such that $\mu\left(x_{n}+K\right)>0$, which implies that $\mu \in A$.

Now assume that $\bar{D} \supset D$. In this case pick $x \in \bar{D}$ and a subsequence $\left(x_{n_{k}}\right)_{k}$ of $\left(x_{n}\right)_{n}$ such that $x_{n_{k}} \rightarrow x$. Set $L=\{x\} \cup\left\{x_{n_{k}}\right\}_{k \geq 1}$ and put $F=\bigcup_{x \in L}(x+K)$. Then $F$ is closed (in fact compact). Note that the corresponding subsequence $\left(\mu_{n_{k}}\right)_{k}$ of $\mu_{n}$ still converges to $\mu$. Arguing as before we conclude that $\mu \in A$ and the proof is complete.

REMARK 2. (a) The fact that $T(A)$ is always dense in $P(X)$ has been proved implicitly by Christensen (see [1] or [3]).

(b) D. Preiss and J. Tišer proved the existence of non-meager Haar-null sets in all separable Banach spaces, with many remarkable additional properties (see [10] or [1]).

(c) Note that part (iii) of Proposition 5 immediately implies that the set of all non-atomic Borel probability measures of $X$ is co-meager in $P(X)$. 
(d) We should point out that the converse implication of part (iii) of Proposition 5 does not hold. For instance, every proper closed subspace of a separable Hilbert space is a Haar-null set (see [1, p. 130]) for which the set of test measures is co-meager. As a matter of fact it can be shown that for every separable reflexive Banach space $X$ and every $F \subseteq X$ weakly closed Haar-null set, the set $T(F)$ is co-meager in $P(X)$.

3. Covering analytic Haar-null sets. In [11], S. Solecki proved that if $A$ is an analytic Haar-null set and $\mu \in P(X)$ is a test measure of $A$, then there exists a Borel Haar-null set $B \supseteq A$, also tested by $\mu$. The aim of this section is to show that the Borel set $B$ may be chosen so that $T(A) \backslash T(B)$ is meager in $P(X)$. In fact we are going to prove the following theorem.

Theorem 6. Let $X$ be an abelian Polish group, $r \geq 0, A \in \Sigma_{1}^{1}(X)$ and $Z \subseteq P(X) \times X$ Borel, such that $\mu(x+A) \leq r$ for every $(\mu, x) \in Z$. Then there exists a Borel set $B \supseteq A$ such that $\mu(x+B) \leq r$ for every $(\mu, x) \in Z$.

Theorem 6 may be proved using the same arguments of Solecki (he used reflection). We will give a different proof of this fact, combinatorial in nature.

Let $Z \subseteq P(X) \times X$ as in Theorem 6 . Enlarge the topology on $P(X) \times X$ to make $Z$ clopen (see [7, p. 82]). Denote this topology by $\tau$. Then $Z$ equipped with the $\tau$ topology becomes a Polish space. Fix a compatible complete metric $d$ for $(Z, \tau)$. Let also $\delta$ be a compatible complete metric for the original topology of $X$. We make the following definition, crucial for the proof.

Definition 7. Let $A \subseteq X, \varepsilon \geq 0$ and $F \subseteq Z$. We will say that $A$ is $(\varepsilon, F)$-covered if there is a Borel set $B \supseteq A$ such that $\mu(x+B) \leq \varepsilon$ for every $(\mu, x) \in F$.

We will need the following two simple lemmas concerning $(\varepsilon, F)$-covered sets. The first one is obvious.

Lemma 8. Let $A \subseteq X, r \geq 0$ and $F \subseteq Z$. Then $A$ is $(r, F)$-covered if and only if $A$ is $(\varepsilon, F)$-covered for every $\varepsilon>r$.

Lemma 9. Let $\varepsilon \geq 0, A \subseteq X$ and $\left(A_{m}\right)_{m}$ a sequence of subsets of $X$ such that $A_{m} \uparrow A$. Let also $F \subseteq Z$ and $\left(F_{n}\right)_{n}$ a sequence of arbitrary subsets of $Z$ such that $F=\bigcup_{n} F_{n}$. Then the following are equivalent:

(i) $A$ is $(\varepsilon, F)$-covered.

(ii) For every $n, m$ the set $A_{m}$ is $\left(\varepsilon, F_{n}\right)$-covered.

Proof. Clearly only the implication (ii) $\Rightarrow$ (i) needs to be proved. So assume that for every $n, m$ the set $A_{m}$ is $\left(\varepsilon, F_{n}\right)$-covered. Pick $B_{n, m} \supseteq A_{m}$ Borel witnessing this fact. Thus $\mu\left(x+B_{n, m}\right) \leq \varepsilon$ for every $(\mu, x) \in F_{n}$. 
Put $C_{m}=\bigcap_{n} \bigcap_{k \geq m} B_{n, k}$. Then $C_{m}$ is Borel and $C_{m} \supseteq A_{m}$ for every $m$, since $B_{n, k} \supseteq A_{k} \supseteq A_{m}$ for every $n$ and every $k \geq m$. In addition we have $\mu\left(x+C_{m}\right) \leq \varepsilon$ for every $(\mu, x) \in F$. As the sequence $\left(C_{m}\right)_{m}$ is increasing, setting $C=\bigcup_{m} C_{m}$ we see that $C$ is Borel, $C \supseteq A$ and $\mu(x+C) \leq \varepsilon$ for every $(\mu, x) \in F$ (the last inequality holds from the continuity of the measure). This implies that $A$ is $(\varepsilon, F)$-covered and the proof is complete.

For every $K \subseteq X$ closed (in the original topology of $X$ ) and every $i \geq 1$, we put $K_{i}=\{x \in X: \delta(x, K) \leq 1 / i\}$ (recall that $\delta$ is the fixed compatible complete metric for $X)$. Note that $K=\bigcap_{i} K_{i}$ and that $K \subseteq \operatorname{Int}\left(K_{i}\right)$ for every $i$ (again the interior is taken in the original topology of $X$ ).

Lemma 10. Let $\left(F_{i}\right)_{i}$ be a decreasing sequence of $\tau$-closed subsets of $Z$ such that d-diam $F_{i} \rightarrow 0$. Let also $K \subseteq X$ be closed (in the original topology) and $(r, Z)$-covered. Then for every $\varepsilon>r$, there exists an $i$ such that $F_{i} \subseteq$ $\left\{(\mu, x): \mu\left(x+K_{i}\right)<\varepsilon\right\}$.

Proof. Suppose not. Then there exists an $\varepsilon>r$ such that for every $i$ we have $F_{i} \not \subseteq\left\{(\mu, x): \mu\left(x+K_{i}\right)<\varepsilon\right\}$ or equivalently $F_{i} \cap\left\{(\mu, x): \mu\left(x+K_{i}\right)\right.$ $\geq \varepsilon\} \neq \emptyset$. We set $L_{i}=F_{i} \cap\left\{(\mu, x): \mu\left(x+K_{i}\right) \geq \varepsilon\right\}$. By Lemma $2(\mathrm{i}), L_{i}$ is a $\tau$-closed subset of $Z$ (recall that the $\tau$ topology on $Z$ is larger than the original topology). Moreover, the sequence $\left(L_{i}\right)_{i}$ is decreasing, as the sequences $\left(F_{i}\right)_{i}$ and $\left(K_{i}\right)_{i}$ are decreasing, and obviously $d$-diam $L_{i} \rightarrow 0$. Hence $\bigcap_{i} L_{i}=\{(\nu, y)\} \in Z$. But then $\nu(y+K)=\lim \nu\left(y+K_{i}\right) \geq \varepsilon>r$, contradicting the fact that $K$ is $(r, Z)$-covered.

We will also need the following representation of analytic sets. For a proof we refer to [7, p. 200].

Theorem 11. Let $X$ be a Polish space and $A$ an analytic subset of $X$. Then there is a regular Suslin scheme $\left(P_{s}\right)_{s \in \mathbb{N}<\mathbb{N}}$ with $A=\mathcal{A}_{s} P_{s}$ such that:

(i) $P_{s}$ is analytic.

(ii) $P_{\emptyset}=A, P_{s}=\bigcup_{n} P_{s^{\urcorner} n}$ and also $P_{s^{\urcorner} n} \subseteq P_{s^{\urcorner} m}$ if $n \leq m$.

(iii) For every $y \in \mathcal{N}, P_{y}=\bigcap_{n} P_{y \mid n}$ is compact.

(iv) If $U \subseteq X$ is open such that $P_{y} \subseteq U$, there there is an $n$ such that $P_{y \mid m} \subseteq U$ for every $m \geq n$.

We are ready to complete the proof of Theorem 6 .

Proof of Theorem 6. Assume that the conclusion of the theorem fails. So, according to our terminology, $A$ is not $(r, Z)$-covered. By Lemma 8 , there exists an $\varepsilon>r$ such that $A$ is not $(\varepsilon, Z)$-covered. Put $Z=\bigcup_{n} F_{n}^{1}$, where each $F_{n}^{1}$ is $\tau$-closed and $d$-diam $F_{n}^{1} \leq 1 / 2$. Let $\left(P_{s}\right)_{s \in \mathbb{N}<\mathbb{N}}$ be the regular Suslin scheme obtained from Theorem 11 for $A$. Then $A=P_{\emptyset}=\bigcup_{m} P_{m}$ with $P_{m} \uparrow A$. As $A$ is not $(\varepsilon, Z)$-covered, by Lemma 9 there exist $n_{1}$ and $m_{1}$ such that $P_{m_{1}}$ is not $\left(\varepsilon, F_{n_{1}}^{1}\right)$-covered. 
Continuing this way, we define (recursively) a $y \in \mathcal{N}$ and a decreasing sequence $Z \supseteq F_{n_{1}}^{1} \supseteq \cdots \supseteq F_{n_{i}}^{i} \supseteq \cdots$ of $\tau$-closed subsets of $Z$ such that for every $i$,

(i) $P_{y \mid i}$ is not $\left(\varepsilon, F_{n_{i}}^{i}\right)$-covered,

(ii) $d$-diam $F_{n_{i}}^{i} \rightarrow 0$.

Put $K=\bigcap_{i} P_{y \mid i}$, which is a compact (hence closed) subset of $A$. So $K$ is $(r, Z)$-covered. Applying Lemma 10 to the sequence $\left(F_{n_{i}}^{i}\right)_{i}$ and $K$, we find that there exists an $i$ such that $F_{n_{i}}^{i} \subseteq\left\{(\mu, x): \mu\left(x+K_{i}\right)<\varepsilon\right\}$. As $K \subseteq \operatorname{Int}\left(K_{i}\right)$, by the adopted representation, for this particular $i$ there is an $n$ such that $P_{y \mid m} \subseteq \operatorname{Int}\left(K_{i}\right)$ for every $m \geq n$. We set $l=\max \{n, i\}$. Then $P_{y \mid l} \subseteq P_{y \mid n} \subseteq \operatorname{Int}\left(K_{i}\right) \subseteq K_{i}$ and moreover $F_{n_{l}}^{l} \subseteq F_{n_{i}}^{i} \subseteq\{(\mu, x)$ : $\left.\mu\left(x+K_{i}\right)<\varepsilon\right\}$. This implies that $P_{y \mid l}$ is $\left(\varepsilon, F_{n_{l}}^{l}\right)$-covered and we derive a contradiction.

A corollary of Theorem 6 is the following.

Corollary 12. Let $A \subseteq X$ be an analytic Haar-null set. Then there exists a Borel Haar-null set $B \supseteq A$ such that $T(A) \backslash T(B)$ is meager in $P(X)$.

Proof. By Lemma 4, $T(A)$ is co-analytic in $P(X)$. So it has the Baire property. Pick $L \subseteq T(A)$ Borel such that $T(A) \backslash L$ is meager in $P(X)$. Put $Z=L \times X$. Then $Z$ is Borel and obviously $\mu(x+A)=0$ for every $(\mu, x) \in Z$. Applying Theorem 6, we get a Borel set $B \supseteq A$ such that $\mu(x+B)=0$ for every $(\mu, x) \in Z$. Clearly $B$ is as desired.

Let us point out that the Borel set $B$ obtained by Corollary 12 need not be a $G_{\delta}$ set even if the group $X$ is locally compact. To see this, it is enough to consider an analytic Haar-null set $A$ such that $A$ is dense in $X$ and $T(A)$ is co-meager (by Proposition 5(iii), such sets do exist). Now note that if $B$ is a $G_{\delta}$ Haar-null set with $B \supseteq A$, then $B$ must be co-meager and so, by Proposition 5(ii), $T(B)$ is meager. Hence $B$ does not satisfy the conclusion of Corollary 12.

Using Theorem 6 we will also derive an approximation type result for analytic sets. We recall that by a result of Solecki (see [11, p. 208]) in every non-locally compact abelian Polish group there exists an analytic set which cannot be approximated up to Haar-null sets by Borel or even co-analytic sets.

Proposition 13. Let $A \subseteq X$ analytic and $\mu \in P(X)$. Then there exist $B \supseteq A$ Borel and $G \subseteq X$ co-meager such that $\mu(x+A)=\mu(x+B)$ for every $x \in G$. 
Proof. Let $n \geq 1$. For every $k=0, \ldots, n+1$ put

$$
F_{k}^{n}=\left\{x \in X: \frac{k}{n+1} \leq \mu(x+A)<\frac{k+1}{n+1}\right\} .
$$

Then observe that $X=\bigcup_{k=0}^{n+1} F_{k}^{n}$. Moreover, it is easy to verify that each $F_{k}^{n}$ is the intersection of an analytic and a co-analytic set. So each $F_{k}^{n}$ has the Baire property. Pick $G_{k}^{n} \subseteq F_{k}^{n}$ Borel such that $F_{k}^{n} \backslash G_{k}^{n}$ is meager. Let $Z_{k}^{n}=\{\mu\} \times G_{k}^{n}$. Then for every $k=0, \ldots, n+1$ the set $Z_{k}^{n}$ is Borel in $P(X) \times X$ and $\mu(x+A) \leq(k+1) /(n+1)$ for every $x \in G_{k}^{n}$, thus for every $(\mu, x) \in Z_{k}^{n}$. Apply Theorem 6 to get a Borel set $B_{k}^{n} \supseteq A$ such that $\mu\left(x+B_{k}^{n}\right) \leq(k+1) /(n+1)$ for every $(\mu, x) \in Z_{k}^{n}$. Put $B_{n}=\bigcap_{k=0}^{n+1} B_{k}^{n}$ and $G_{n}=\bigcup_{k=0}^{n+1} G_{k}^{n}$. Then $G_{n} \subseteq X$ is co-meager, $B_{n} \supseteq A$ is Borel and

$$
\mu\left(x+B_{n}\right)-\mu(x+A) \leq \frac{1}{n+1}
$$

for every $x \in G_{n}$. Finally, let $B=\bigcap_{n} B_{n}$ and $G=\bigcap_{n} G_{n}$. Clearly $B$ and $G$ are as desired.

4. Results under Martin's axiom. The aim of this section is to show that under some stronger set-theoretic assumptions, in particular under Martin's Axiom and the negation of Continuum Hypothesis (abbreviated as MA $+\neg \mathrm{CH}$ ), $\boldsymbol{\Pi}_{2}^{1}$ sets satisfy the conclusion of Proposition 13. As before, $X$ is an abelian Polish group. For the following proposition let us recall that if $\left(A_{\xi}\right)_{\xi<\omega_{1}}$ is a transfinite sequence of universally measurable subsets of $X$, then under MA $+\neg \mathrm{CH}$ the set $\bigcap_{\xi<\omega_{1}} A_{\xi}$ is universally measurable (see [8] for a proof).

Proposition 14. (MA $+\neg \mathrm{CH})$ Let $A \in \Pi_{2}^{1}(X)$ and $\mu \in P(X)$ be such that $\mu(x+A)=0$ for every $x \in G$, where $G$ is a co-meager subset of $X$. Then there exist a Borel set $B \supseteq A$ and a co-meager set $G^{\prime} \subseteq X$ such that $\mu(x+B)=0$ for every $x \in G^{\prime}$.

Proof. By a classical result of Sierpiński (see [7, p. 324]), there exists a decreasing transfinite sequence $\left(B_{\xi}\right)_{\xi<\omega_{1}}$ of Borel sets such that $A=$ $\bigcap_{\xi<\omega_{1}} B_{\xi}$. Let $C=\bigcap_{\xi<\omega_{1}} C_{\xi}$, where $C_{\xi}=\left\{x \in X: \mu\left(x+B_{\xi}\right)>0\right\}$. By assumption there is $\xi_{0}<\omega_{1}$ with $C_{\xi_{0}} \backslash C$ meager. Moreover, for each $x \in G$ there is $\lambda<\omega_{1}$ with $\mu\left(x+B_{\lambda}\right)=0$, hence $G \cap C=\emptyset$. It follows that $C$ is meager and therefore so is $C_{\xi_{0}}$. We set $B=B_{\xi_{0}}$ and $G^{\prime}=X \backslash C_{\xi_{0}}$.

Note that the Borel set $B$ obtained from Proposition 14 need not be Haar-null even if its translates are $\mu$-null over a large set (in the topological sense). This phenomenon does not occur if co-meagerness is replaced by prevalence, as the following proposition shows. 
Proposition 15. Let $\mu \in P(X)$ and $A, G \subseteq X$ be universally measurable sets such that $\mu(x+A)=0$ for every $x \in G$. If $G$ is prevalent, then $A$ is Haar-null.

Proof. Note that $-G=\{-x: x \in G\}$ is also prevalent. Let $\nu \in P(X)$ be a test measure for $-G$. So $\nu(x-G)=1$ for every $x \in X$. Then we observe that

$$
\begin{aligned}
(\nu * \mu)(x+A) & =\int_{X} \mu(x+A-y) d \nu(y) \\
& =\int_{x-G} \mu(x+A-y) d \nu(y)+\int_{X \backslash x-G} \mu(x+A-y) d \nu(y) \\
& \leq \nu(X \backslash x-G)=1-\nu(x-G)=0
\end{aligned}
$$

for every $x \in X$. So $A$ is Haar-null and $\nu * \mu$ is a test measure for $A$.

We will also show that if $A \subseteq X$ is analytic or co-analytic, then there exists a Borel set $B \subseteq A$ such that $T(B) \backslash T(A)$ is meager in $P(X)$. Actually, for co-analytic sets one does not need the full strength of $\mathrm{MA}+\neg \mathrm{CH}$, but only the statement that "all $\boldsymbol{\Sigma}_{2}^{1}$ sets have the Baire property" (for the relationship between these statements see [6]). The following proposition has been suggested to us by the referee.

Proposition 16. Assume that all $\boldsymbol{\Sigma}_{2}^{1}$ sets have the Baire property. Then for any co-analytic set $A$, there exists a Borel set $B \subseteq A$ such that $T(B) \backslash$ $T(A)$ is meager in $P(X)$.

Proof. Let WO $\subseteq 2^{\mathbb{N}^{2}}$ be the set of all well-orderings of $\mathbb{N}$. For $\gamma \in \mathrm{WO}$, $|\gamma|$ stands for the ordinal isomorphic to the well-ordering coded by $\gamma$. The set WO is $\boldsymbol{\Pi}_{1}^{1}$-complete and the map $\gamma \mapsto|\gamma|$ is a $\boldsymbol{\Pi}_{1}^{1}$-rank for WO (see [7, pp. 213 and 267]). So there is a Borel function $f: X \rightarrow 2^{\mathbb{N}^{2}}$ such that $x \in A$ iff $f(x) \in$ WO. Let $\phi: A \rightarrow \omega_{1}$ be defined by $\phi(x)=|f(x)|$. Then $\phi$ is a $\Pi_{1}^{1}$-rank for $A$. For every countable ordinal $\xi$, set as usual $A_{\xi}=\{x \in A: \phi(x) \leq \xi\}$ and note that each $A_{\xi}$ is Borel and $A=\bigcup_{\xi<\omega_{1}} A_{\xi}$.

Claim. We have $T(A)=\bigcap_{\xi<\omega_{1}} T\left(A_{\xi}\right)$.

Proof. It is clear that $T(A) \subseteq \bigcap_{\xi<\omega_{1}} T\left(A_{\xi}\right)$. To show the other inclusion, let $\mu \in \bigcap_{\xi<\omega_{1}} T\left(A_{\xi}\right)$. Let $x \in X$ arbitrary. From the regularity of the measure $\mu$, there exists a Borel set $B \subseteq A$ with $\mu(x+A)=\mu(x+B)$. As $B \subseteq A$ is Borel, by the Boundedness Theorem for $\boldsymbol{\Pi}_{1}^{1}$-ranks (see [7, p. 288]), it follows that $\sup \{\phi(x): x \in B\}=\lambda<\omega_{1}$ and so $B \subseteq A_{\lambda} \subseteq A$. From the fact that $\mu \in T\left(A_{\lambda}\right)$, we get

$$
\mu(x+A)=\mu(x+B)=\mu\left(x+A_{\lambda}\right)=0 .
$$

As $x$ was arbitrary, we conclude that $\mu \in T(A)$ as desired. 
Consider the relation

$$
\begin{aligned}
R=\left\{(x, y, \gamma) \in X \times X \times 2^{\mathbb{N}^{2}}\right. & : \gamma \notin \mathrm{WO} \text { or } \\
& (f(x-y) \in \mathrm{WO} \text { and }|f(x-y)| \leq|\gamma|)\} .
\end{aligned}
$$

As the relation

$$
\gamma \notin \mathrm{WO} \text { or }(\delta \in \mathrm{WO} \text { and }|\delta| \leq|\gamma|)
$$

is $\boldsymbol{\Sigma}_{1}^{1}$ on $(\delta, \gamma) \in 2^{\mathbb{N}^{2}} \times 2^{\mathbb{N}^{2}}$ (see [7, p. 269]), it follows that $R$ is $\boldsymbol{\Sigma}_{1}^{1}$. Furthermore, if $\gamma \in \mathrm{WO}$ and $y \in X$, for the section $R_{(y, \gamma)}=\{x \in X:(x, y, \gamma) \in R\}$ we have

$$
R_{(y, \gamma)}=y+A_{|\gamma|}
$$

For $\mu, \nu \in P(X)$ define

$$
\begin{aligned}
\mu \leq^{*} \nu \Leftrightarrow & (\mu, \nu \notin T(A) \text { and } \\
& \left.\forall \gamma \in 2^{\mathbb{N}^{2}}\left[\forall y \in X \nu\left(R_{(y, \gamma)}\right)=0 \Rightarrow \forall y \in X \mu\left(R_{(y, \gamma)}\right)=0\right]\right) .
\end{aligned}
$$

The set $P(X) \backslash T(A)$ is $\boldsymbol{\Sigma}_{2}^{1}$. Moreover, since $R$ is $\boldsymbol{\Sigma}_{1}^{1}$, the rest of the right hand side of the above formula is $\Pi_{2}^{1}$ by Theorem 29.26 in [7]. Thus, by our assumption, $\leq^{*} \subseteq P(X) \times P(X)$ has the Baire property in $P(X) \times P(X)$. Moreover, by (1), for $\mu, \nu \notin T(A), \mu \leq^{*} \nu$ is equivalent to saying that for each $\gamma \in \mathrm{WO}$,

$$
\nu \in T\left(A_{|\gamma|}\right) \Rightarrow \mu \in T\left(A_{|\gamma|}\right)
$$

that is,

$$
\mu \leq^{*} \nu \Leftrightarrow \xi_{\mu} \leq \xi_{\nu}
$$

where $\xi_{\mu}=\min \left\{\xi<\omega_{1}: \mu \notin T\left(A_{\xi}\right)\right\}$ and similarly for $\xi_{\nu}$. As $\left(T\left(A_{\xi}\right)\right)_{\xi<\omega_{1}}$ is a decreasing transfinite sequence of sets with the Baire property, there is $\xi_{0}<\omega_{1}$ such that for all $\xi \geq \xi_{0}$ the set $T\left(A_{\xi_{0}}\right) \backslash T\left(A_{\xi}\right)$ is meager. By Exercise 8.49 in [7], applied to $\leq^{*}$ on $T\left(A_{\xi_{0}}\right) \backslash T(A)$, we deduce that

$$
T\left(A_{\xi_{0}}\right) \backslash T(A)=\bigcup_{\xi_{0} \leq \xi<\omega_{1}}\left(T\left(A_{\xi_{0}}\right) \backslash T\left(A_{\xi}\right)\right)
$$

is meager and the proof is complete.

Under MA $+\neg \mathrm{CH}$, the above result is also valid for analytic sets. First we need the following extension of Lemma 3. Again the proof is omitted.

LEMMA 17. (MA $+\neg \mathrm{CH})$ Let $\left(A_{\xi}\right)_{\xi<\omega_{1}}$ be an increasing transfinite sequence of Borel sets. Then $T(A)=\bigcap_{\xi<\omega_{1}} T\left(A_{\xi}\right)$, where $A=\bigcup_{\xi<\omega_{1}} A_{\xi}$. In particular $T(A)$ has the Baire property.

We also need the following. Its proof is left to the reader.

Lemma 18. (MA $+\neg \mathrm{CH})$ Let $\left(A_{\xi}\right)_{\xi<\omega_{1}}$ and $A$ be as in Lemma 17. Then there exists a countable ordinal $\xi$ such that $T\left(A_{\xi}\right) \backslash T(A)$ is meager in $P(X)$. 
Combining Lemma 18 with Sierpiński's result, we get the following.

Proposition 19. (MA $+\neg \mathrm{CH})$ Let $A \subseteq X$ be analytic. Then there exists a Borel set $B \subseteq A$ such that $T(B) \backslash T(A)$ is meager in $P(X)$.

Remark 3. In [4], R. Dougherty asked whether any analytic non Haarnull set must include a Borel non-Haar-null set. By Proposition 19, under $\mathrm{MA}+\neg \mathrm{CH}$ any analytic non-Haar-null set $A$ includes a Borel set $B$ such that $T(B)$ is meager in $P(X)$. That is, the set $B$ may be Haar-null, but only a relatively small set of measures witness this fact. We should point out that not every universally measurable non-Haar-null set shares this property. For instance, Dougherty considered a set $A \subseteq X$ such that $|A \cap B|<2^{\aleph_{0}}$ whenever $B$ is a $\sigma$-compact set and $|A \cap B|=2^{\aleph_{0}}$ whenever $B$ is a Borel set not included in a $\sigma$-compact set (see [4, p. 85]). Under Martin's Axiom, this set $A$ is universally measurable and Haar-null. So its complement is prevalent. However, if $B$ is any Borel set included in $X \backslash A$, then $B$ must be included in a $\sigma$-compact set. Hence, by Proposition 5(iii), $T(B)$ must be co-meager in $P(X)$.

Let us conclude this section with some remarks concerning a possible extension of Corollary 12 to co-analytic sets. To the best of our knowledge, even the problem whether for any co-analytic Haar-null set $A \subseteq X$ there exists a Borel Haar-null set $B \supseteq A$, is open. In this direction it seems quite natural to ask whether under $\mathrm{MA}+\neg \mathrm{CH}$ the conclusion of Corollary 12 is valid for co-analytic sets.

5. An application. Let $X$ be a separable Banach space. A locally Lipschitz function $f: X \rightarrow \mathbb{R}$ is said to be an essentially smooth Lipschitz function if the set

$$
S_{f}=\{x \in X: \partial f(x) \text { is not a singleton }\}
$$

is contained in the $\sigma$-ideal generated by the Borel Haar-null sets (by $\partial f$ we denote the subdifferential of $f$ in the sense of Clarke). That is, $f$ is said to be essentially smooth if there exists a Borel Haar-null set $B \subseteq X$ such that $B \supseteq S_{f}$. The class of essentially smooth Lipschitz functions was defined and studied extensively by J. M. Borwein and W. B. Moors in [2]. They proved that the members of this class possess differentiability properties similar to the ones of convex functions (for instance their Clarke subdifferential is both minimal and integrable). In addition they showed that this class has very strong stability properties (it is closed under addition, multiplication and lattice operations).

Note however that the set $S_{f}$ is not Borel in general. So it might belong to the $\sigma$-ideal of universally measurable Haar-null sets but not to the $\sigma$-ideal generated by the Borel Haar-null sets (as we have already mentioned, the 
existence of such sets was observed by Dougherty). We will show that in this particular situation there is no difference.

Proposition 20. If the set $S_{f}$ is contained in the $\sigma$-ideal of universally measurable Haar-null sets, then there exists a Borel Haar-null set $B \subseteq X$ such that $B \supseteq S_{f}$.

Proof. First of all observe that since $X$ is a separable Banach space, the space $\left(X^{*}, B\left(X_{w^{*}}^{*}\right)\right)$, i.e. the Borel $\sigma$-algebra of the weak ${ }^{*}$ topology of $X^{*}$, is a standard Borel space (see [7, p. 79]). So fix a Polish topology $\tau$ on $X^{*}$ such that $B\left(X_{\tau}^{*}\right)=B\left(X_{w^{*}}^{*}\right)$. From the fact that $\partial f$ has closed graph in $X \times X_{w^{*}}^{*}$ we get

$$
\operatorname{Gr} \partial f \in B(X) \otimes B\left(X_{w^{*}}^{*}\right)=B(X) \otimes B\left(X_{\tau}^{*}\right)=B\left(X \times X_{\tau}^{*}\right) .
$$

As $\operatorname{Gr} \partial f$ is a Borel subset of $X \times X_{\tau}^{*}$, it admits a co-analytic uniformization $A \subseteq X \times X_{\tau}^{*}($ see $[7$, p. 306]). But then observe that

$$
S_{f}=\operatorname{proj}_{X}\left\{\operatorname{Gr} \partial f \cap\left(\left(X \times X^{*}\right) \backslash A\right)\right\} .
$$

Clearly the set $S_{f}$ is analytic. So if $S_{f}$ is Haar-null, by Corollary 12, there exists a Borel Haar-null set $B \subseteq X$ such that $S_{f} \subseteq B$.

Acknowledgments. I would like to thank the anonymous referee for his thorough report, which substantially improved the presentation of the paper.

\section{References}

[1] Y. Benyamini and J. Lindenstrauss, Geometric Nonlinear Functional Analysis, Amer. Math. Soc. Colloq. Publ. 48, Amer. Math. Soc., 2000.

[2] J. M. Borwein and W. B. Moors, Essentially smooth Lipschitz functions, J. Funct. Anal. 149 (1997), 305-351.

[3] J. P. R. Christensen, On sets of Haar measure zero in abelian Polish groups, Israel J. Math. 13 (1972), 255-260.

[4] R. Dougherty, Examples of non-shy sets, Fund. Math. 144 (1994), 73-88.

[5] B. Hunt, T. Sauer and J. Yorke, Prevalence: a translation-invariant "almost every" on infinite-dimensional spaces, Bull. Amer. Math. Soc. 27 (1992), 217-238.

[6] A. Kanamori, The Higher Infinite: Large Cardinals in Set Theory, Springer Monogr. Math., Springer, 2003.

[7] A. S. Kechris, Classical Descriptive Set Theory, Grad. Texts in Math. 156, Springer, 1995.

[8] K. Kunen, Set Theory, North-Holland, 1980.

[9] J. Mycielski, Some unsolved problems on the prevalence of ergodicity, instability and algebraic independence, Ulam Quart. 1 (1992), no. 3, 30-37.

[10] D. Preiss and J. Tišer, Two unexpected examples concerning differentiability of Lipschitz functions on Banach spaces, in: Geometric Aspects of Functional Analysis (Israel, 1992-1994), Birkhäuser, 1995, 219-238. 
[11] S. Solecki, On Haar null sets, Fund. Math. 149 (1996), 205-210.

[12] —, Haar-null and non-dominating sets, ibid. 170 (2001), 197-217.

Department of Mathematics

National Technical University of Athens

Zografou Campus, 15780 Athens, Greece

E-mail: pdodos@math.ntua.gr

Received 16 September 2002;

in revised form 8 March 2004 